Electron-Heated Target Temperature Measurements in Petawatt Laser Experiments Based on Extreme Ultraviolet Imaging and Spectroscopy

T.A. Ma, F.N. Beg, A.G. Macphee, H.-K. Chung, M.H. Key, A.J. Mackinnon, P.K. Patel, S.P. Hatchett, K.U. Akli, R.B. Stephens, C.D. Chen, R.R. Freeman, A. Link, D.T. Offermann, V. Ovchinnikov, L.D. VanWoerkom, B. Zhang

May 5, 2008

High Temperature Plasma Diagnostics 2008 Albuquerque, NM, United States May 11, 2008 through May 15, 2008 
This document was prepared as an account of work sponsored by an agency of the United States government. Neither the United States government nor Lawrence Livermore National Security, LLC, nor any of their employees makes any warranty, expressed or implied, or assumes any legal liability or responsibility for the accuracy, completeness, or usefulness of any information, apparatus, product, or process disclosed, or represents that its use would not infringe privately owned rights. Reference herein to any specific commercial product, process, or service by trade name, trademark, manufacturer, or otherwise does not necessarily constitute or imply its endorsement, recommendation, or favoring by the United States government or Lawrence Livermore National Security, LLC. The views and opinions of authors expressed herein do not necessarily state or reflect those of the United States government or Lawrence Livermore National Security, LLC, and shall not be used for advertising or product endorsement purposes. 


\title{
Electron heated target temperature measurements in petawatt laser experiments based on extreme ultraviolet imaging and spectroscopy
}

\author{
T. Ma and F. N. Beg \\ Department of Mechanical and Aerospace Engineering, University of California-San \\ Diego, La Jolla, CA \\ A. G. MacPhee, H.-K. Chung, M. H. Key, A. J. Mackinnon, P. K. Patel, and S. Hatchett \\ Lawrence Livermore National Laboratory, Livermore, CA \\ K. U. Akli and R. B. Stephens \\ General Atomics, San Diego, CA \\ C. D. Chen \\ Plasma Science Fusion Center, Massachusetts Institute of Technology, Cambridge, \\ Massachusetts \\ R. R. Freeman, A. Link, D. T. Offermann, V. Ovchinnikov, and L. D. Van Woerkom \\ College of Mathematical and Physical Sciences, The Ohio State University, Columbus, \\ $\mathrm{OH}$ \\ B. Zhang \\ Department of Applied Science, University of California-Davis, Davis, CA
}

\begin{abstract}
Three independent methods (XUV spectroscopy, imaging at $68 \mathrm{eV}$ and $256 \mathrm{eV}$ ) have been used to measure planar target rear surface plasma temperature due to heating by hot electrons. The hot electrons are produced by ultra-intense laser plasma interactions using the $150 \mathrm{~J}, 0.5 \mathrm{ps}$ Titan laser. Soft x-ray spectroscopy in the $50-400 \mathrm{eV}$ region and imaging at the $68 \mathrm{eV}$ and $256 \mathrm{eV}$ photon energies were used to determine the rear surface temperature of planar CD targets. Temperatures were found to be in the $60-150 \mathrm{eV}$ range, with good agreement between the three diagnostics.
\end{abstract}

\section{Introduction}

The use of soft x-ray imaging and spectroscopy can be an incredibly useful tool to measure the patterns and magnitudes of energy deposition by thermal electrons in laserplasma interactions. ${ }^{1,2,3}$ When an ultra-intense laser hits a solid target, a large flux of energetic electrons are created which then heat the material, resulting in a thermal Planckian emission spectrum peaking in the extreme ultraviolet (XUV) to soft x-ray spectral region. This radiation intensity varies rapidly with temperature, and thus timeintegrated imaging, as well as spectroscopic resolution of the radiation offers an excellent tool to determine plasma temperatures. The aim of this work is to confirm temperatures derived from the XUV imagers against those obtained from the Flat Field Spectrometer (FFS) under short-pulse, high intensity laser irradiation conditions. 


\section{Experiment}

Experiments were carried out on the Titan facility at the Lawrence Livermore National Laboratory. The $150 \mathrm{~J}, 0.5 \mathrm{ps}, \lambda=1053 \mathrm{~nm}$ laser is used to illuminate $25 \mathrm{um}$ thick, $1 \mathrm{~mm} \times 1 \mathrm{~mm}$ deuterated carbon (CD) plastic planar targets under a variety of irradiation conditions (varying on-target laser intensities by changing the focal spot size). The Planckian radiation emitted when the target heats up is captured by a Flat Field Spectrometer $^{3}$ (FFS) which records spectra in the $50-400 \mathrm{eV}(30-250 \mathrm{~A}$ wavelength) range and two XUV imagers ${ }^{1}$, one operating at the $68 \mathrm{eV}$ photon energy, the other at the $256 \mathrm{eV}$ photon energy. The experimental setup is illustrated in Fig.1.

The FFS consists of a 1200 lines/mm concave diffraction grating set to a glancing angle of $4.6^{\circ}$. To increase the flux density at the detector plane, a cylindrical Au mirror is set at a glancing angle of $4^{\circ}$, with its surface orthogonal to the flat-field grating. The detector is a vacuum charge coupled device (CCD) detector (back thinned 1340 x 1300 array with $20 \mu \mathrm{m}$ pixel size) in a circular detector plane. A limited aperture filter between the grating and detector discriminates against fluorescent and scattered emission within the spectrometer. The diffraction grating is located at a distance of $1.25 \mathrm{~m}$ from the target being imaged, and the detector plane is $0.235 \mathrm{~m}$ from the grating center. The view angle was $34^{\circ}$ with respect to target rear side normal. The solid subtended by the spectrometer was $5.69 \times 10^{-5} \mathrm{Sr}$ and tests demonstrated a spatial resolution of $50 \mu \mathrm{m}$.

XUV imaging at two photon energies (i.e., 68 and $256 \mathrm{eV}$ ) is used. Each of the XUV imagers consists of a spherical multilayer mirror ${ }^{5}$ that focuses the target rear surface emission onto a flat mirror, which turns the beam through $90^{\circ}$. Each spherical-plane mirror pair has matching spectral peaks and is optimized to image within a specific energy bandwidth. The $68 \mathrm{eV}$ mirrors are a $\mathrm{Mo}_{2} \mathrm{C} / \mathrm{Si}$ multilayer, while the $256 \mathrm{eV}$ mirrors are a $\mathrm{C} / \mathrm{WC} / \mathrm{Monel} / \mathrm{W}$ formula. The spherical mirrors, which have a radius of curvature of $0.5 \mathrm{~m}$, are placed approximately $27 \mathrm{~cm}$ away from the target, and are set to nearnormal incidence. The reflected image is then deflected off the plane mirror and goes through a thin aluminum/polyimide filter, before reaching the vacuum charge coupled device (CCD) detector (back thinned 1340 x 1300 array with $20 \mu \mathrm{m}$ pixel size) at a path length of $2.15 \mathrm{~m}$. The total magnification of the system is approximately 11 and the resolution is approximately $9 \mu \mathrm{m}$. Both XUV diagnostics image the target rear side at a $43^{\circ}$ viewing angle with respect to target normal. The details of the XUV imaging can be found in Ref. 2.

\section{Results and Discussion}

The use of the cylindrical mirror in conjunction with the grating in the FFS allows for both spatial imaging and spectral imaging/dispersion. Indeed, for most targets, a double spectra - one originating from the target rear surface, and one from the front plasma plume is seen in the Flat Field data. The two spectra are slightly shifted relative to each other, likely due to a different hot spot height from the front to the back side, and the emission spectra are generally super-imposed on top of a bremsstrahlung continua. The spectral lines from each side of the target are identified and their relative intensities 
determined. Fig. 2 shows a single shot target front and rear side carbon spectrum (recorded with no low-pass filter).

The atomic spectroscopy code $\mathrm{FLYCHK}^{6}$ is used to calculate line profiles of atomic transitions. FLYCHK uses a detailed level structure for lithium-like, helium-like and hydrogenic ion stages to calculate charge state and population distributions. In our simulations, spectra for densities between $n_{\mathrm{e}}=1 \times 10^{18}-1 \times 10^{21} \mathrm{~cm}^{-3}$ and temperatures from $10-1000 \mathrm{eV}$ are generated under an initial steady-state LTE approximation and a steady state LTE evolution. The steady-state LTE solutions are assumed to allow a single temperature and pressure to be attributed to the whole system for the sake of simplifying the problem. Line pair ratios of intensities of selected transitions are determined from the synthetic spectra and then compared against the experimental spectra.

The rear surface spectra of a $25 \mu \mathrm{m}$ CD planar target irradiated at $144 \mathrm{~J}, 0.5 \mathrm{ps}$, with a $50 \mu \mathrm{m}$ focal spot is analyzed. From the FFS, the experimental ratio of the CVI (hydrogen-like carbon) $4 \mathrm{p}-2 \mathrm{~s}(\lambda=135 \mathrm{~A})$ to $\mathrm{CV}$ (helium-like carbon) $4 \mathrm{p}-2 \mathrm{~s}(\lambda=173 \mathrm{~A})$ is 0.3 ; CVI $3 p-2 s$ ( $\lambda=182 \mathrm{~A})$ to CV $4 \mathrm{~d}-2 \mathrm{p}(\lambda=187 \mathrm{~A})$ is 2.5 ; and CVI $3 \mathrm{p}-2 \mathrm{~s}$ to CV $5 \mathrm{~d}-2 \mathrm{p}$ $(\lambda=167 \mathrm{~A})$ is 7.1 . After comparison to synthetic spectra for plasma density $n_{e}=1 \times 10^{18}$, this predicts a plasma temperature of $67 \mathrm{eV}, 76 \mathrm{eV}$, and $80 \mathrm{eV}$ from each of the line pair ratios, respectively. Fig. 3 compares the predicted line ratios with the experimental results.

Imaging using the XUV diagnostic offers a dual function: a qualitative high spatial resolution ( $\sim 9 \mathrm{um})$ visual of the target heating, and a quantitative measurement of the target temperature. Furthermore, the two different XUV energies offers different views of emission: the $68 \mathrm{eV}$ XUV emission captures the long afterglow of the hydrodynamic expansion of the plasma, whereas the $256 \mathrm{eV}$ emission is a much shorter duration glow that "freezes" the hydrodynamic expansion of the exploding target. Examples of raw XUV images for planar targets, and the subsequent analysis of them to convert to absolute XUV brightnesses and temperature maps are presented in Ref. 2.

A peak temperature of $127 \mathrm{eV}$ and $154 \mathrm{eV}$ are obtained from the spatially resolved 68 $\mathrm{eV}$ and $256 \mathrm{eV} \mathrm{XUV}$ images, respectively, for the same CD target as analyzed using the FFS. These temperatures are in agreement to within 5\%.

The temperatures calculated from the two XUV imagers, while fairly consistent with each other, give temperatures approximately $70 \mathrm{eV}$ higher than the temperature predicted by the FFS (nearly a factor of 2). The XUV has errors associated with applying a 2-D model to an actual 3-D expansion, as well as analytic fitting to the model. With the FFS, there are errors associated with continuum corrections and blends with neighboring lines when determining the integrated line intensities. The determination of temperature in this case came from comparing line ratios with expected spectra at a plasma density of $10^{18}$ $\mathrm{cm}^{-3}$-- only a rough estimate of the density of the plasma plume. Furthermore, the steady-state LTE approximation only serves to give a rough picture of the population distributions. In reality, both temperature and the density of the plasma are temporally evolving, and interpolation schemes and opacity corrections need to be applied.

To conclude, we have made a comparison of plasma temperature at the rear surface of the target using three methods. The temperatures obtained are consistent with each technique having errors. This comparison provides confidence in each diagnostic used.

\section{Acknowledgments}


The authors thank the Jupiter Laser Facility staff. This work was performed under the auspices of the U.S. Department of Energy by Lawrence Livermore National Laboratory under contracts DE-FG02-05ER54834, W-7405-Eng-48 No. DE-FC02-04ER54789 (Fusion Science Center) and DE-AC52-07NA27344. T. Ma is funded through LLNL's Institute of Laser Science and Applications grant.

${ }^{1}$ P. Gu et al., Rev. Sci. Instrum. 77, 113101 (2006).

${ }^{2}$ T. Ma et al., Rev. Sci. Instrum. manuscript submitted

${ }^{3}$ T. Tokushima et al., Surf. Rev. Lett. 9, 503-508 (2002) .

${ }^{4}$ D. Neely et al., CLF Annual Report 1994-1995.

${ }^{5}$ T. W. Barbee, Jr., Opt. Eng. 25 (8), 898-915 (1986).

${ }^{6}$ H.-K. Chung, et al., High Energy Density Phys. 1, 3 (2005).

\section{Figure Captions}

Fig. 1: Schematic of the experimental setup.

Fig. 2: (a) The soft $\mathrm{x}$-ray spectra for a CD planar target using a 1200 lines/mm diffraction grating. (b) The lineouts for the front and rear surface spectra.

Fig. 3: The comparison of measured experimental line pair ratios with expected line pair ratios generated using the FLYCHK spectroscopic code. 


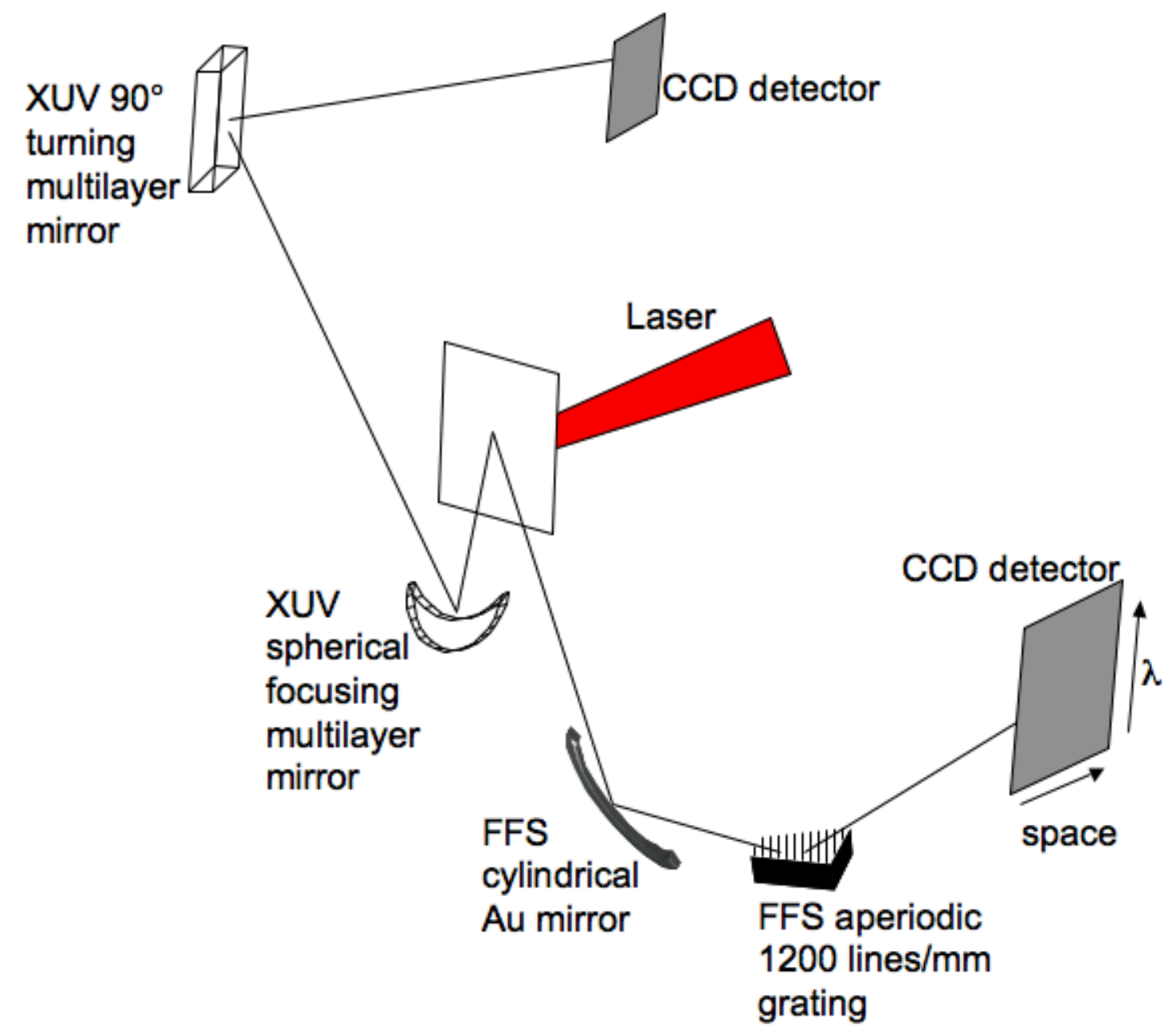

Fig. 1. 
(a)

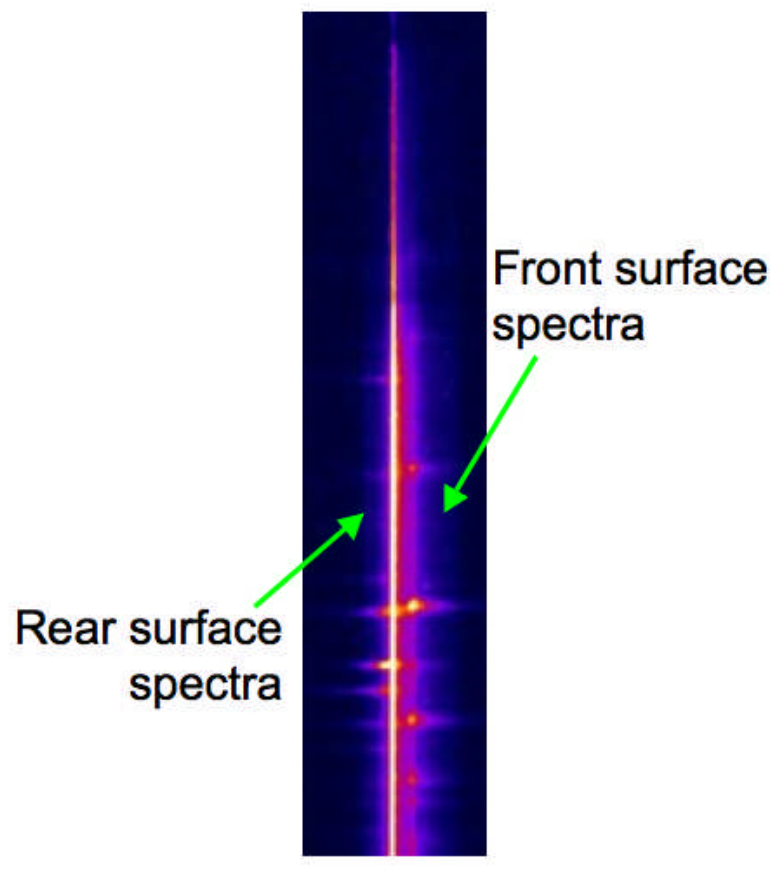

(b)

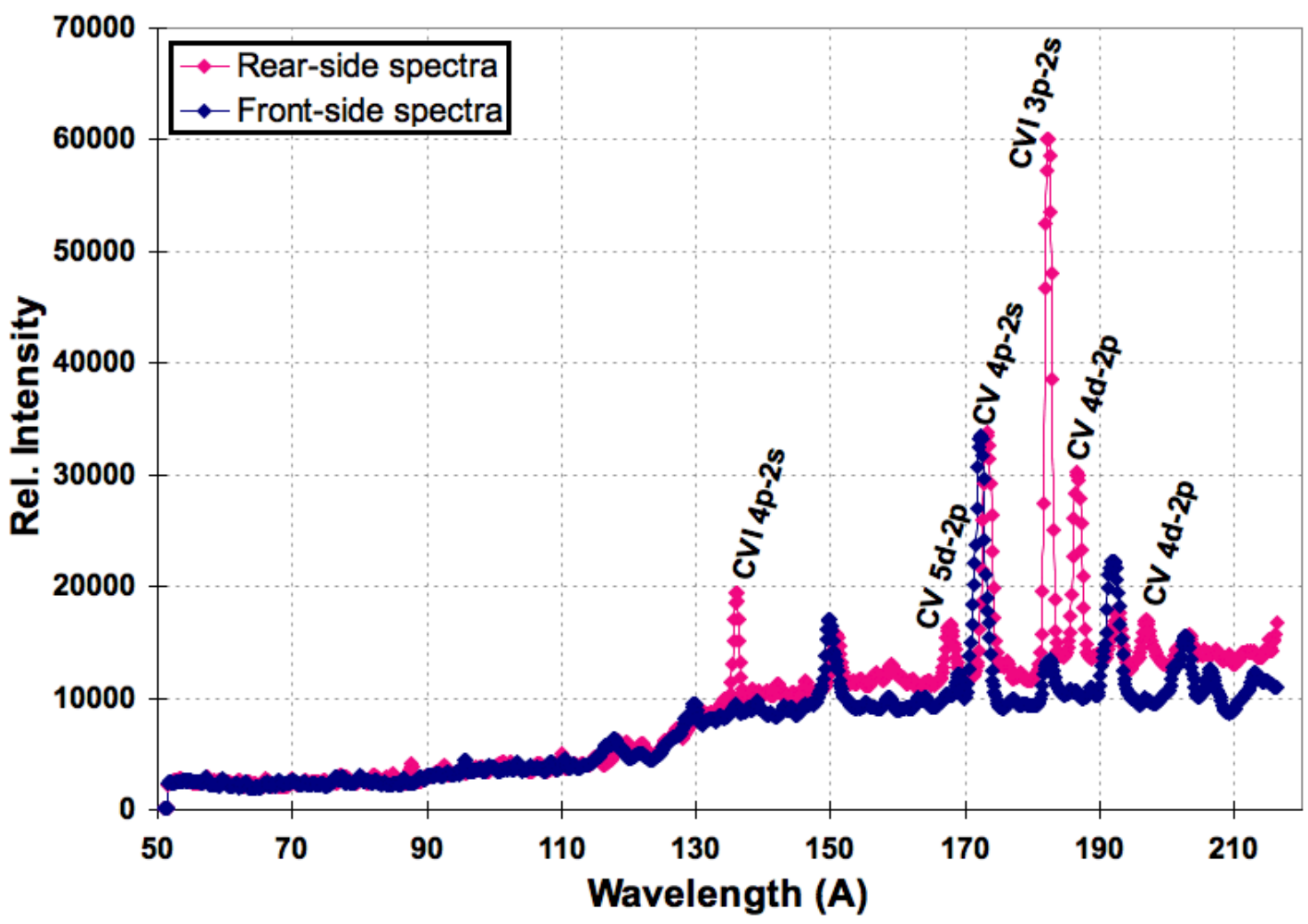

Fig. 2 


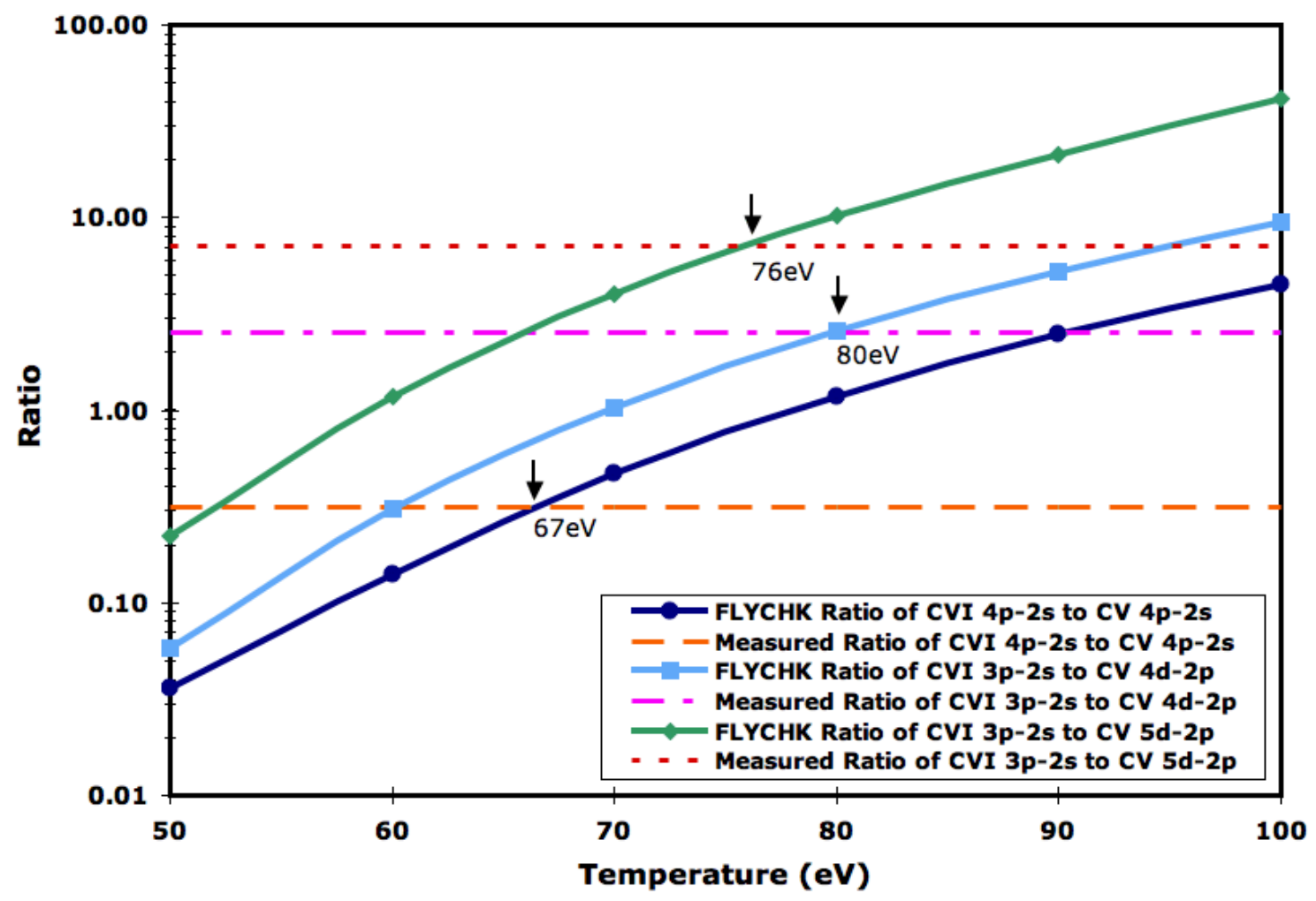

Fig. 3 